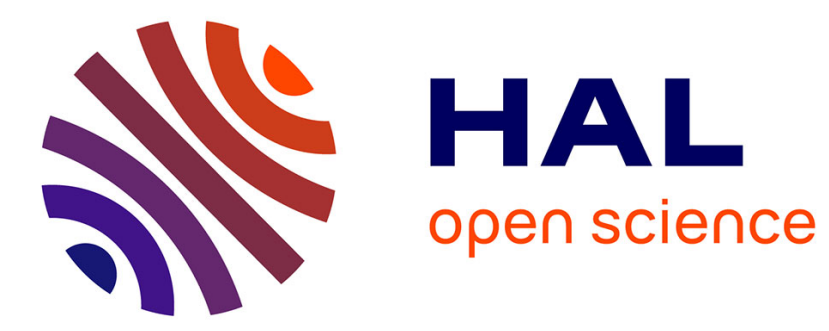

\title{
TssA: The cap protein of the Type VI secretion system tail
}

\author{
Abdelrahim Zoued, Eric Durand, Yoann G Santin, Laure Journet, Alain \\ Roussel, Christian Cambillau, E. Cascales
}

\section{- To cite this version:}

Abdelrahim Zoued, Eric Durand, Yoann G Santin, Laure Journet, Alain Roussel, et al.. TssA: The cap protein of the Type VI secretion system tail. BioEssays, 2017, 39 (10), 10.1002/bies.201600262 . hal-01780742

\section{HAL Id: hal-01780742 \\ https://hal-amu.archives-ouvertes.fr/hal-01780742}

Submitted on 27 Apr 2018

HAL is a multi-disciplinary open access archive for the deposit and dissemination of scientific research documents, whether they are published or not. The documents may come from teaching and research institutions in France or abroad, or from public or private research centers.
L'archive ouverte pluridisciplinaire HAL, est destinée au dépôt et à la diffusion de documents scientifiques de niveau recherche, publiés ou non, émanant des établissements d'enseignement et de recherche français ou étrangers, des laboratoires publics ou privés. 
Abdelrahim Zoued ${ }^{1, \dagger}$, Eric Durand ${ }^{1}$, Yoann G. Santin ${ }^{1}$, Laure Journet ${ }^{1}$, Alain Roussel ${ }^{2,3}$,

8 Running head: T6SS biogenesis

${ }^{1}$ Laboratoire d'Ingénierie des Systèmes Macromoléculaires (LISM), Institut de Microbiologie de la 13 Méditerranée (IMM), CNRS - Aix-Marseille Université UMR7255, 31 chemin Joseph Aiguier, 13402 14 Marseille Cedex 20, France.

2 Architecture et Fonction des Macromolécules Biologiques, Centre National de la Recherche 16 Scientifique, UMR 7257, Campus de Luminy, Case 932, 13288 Marseille Cedex 09, France

$17{ }^{3}$ Architecture et Fonction des Macromolécules Biologiques, Aix-Marseille Université, UMR 7257, 18 Campus de Luminy, Case 932, 13288 Marseille Cedex 09, France

* To whom correspondence should be addressed. E-mail: cascales@imm.cnrs.fr or $21 \quad$ christian.cambillau@afmb.univ-mrs.fr

${ }^{\dagger}$ Current address: Howard Hughes Medical Institute, Brigham and Women's Hospital, Division of 24 Infectious Diseases and Harvard Medical School, Department of Microbiology and Immunology, Boston, Massachusetts, USA

Word count (excluding Figure legends and references: 20,000)

Table: 1

Figures: 7 
3 The Type VI secretion system (T6SS) is a multiprotein and mosaic apparatus that delivers 4 protein effectors into prokaryotic or eukaryotic cells. Recent data on the enteroaggregative 5 Escherichia coli (EAEC) T6SS have provided evidence that the TssA protein is a key 6 component during T6SS biogenesis. The T6SS comprises a trans-envelope complex that 7 docks the baseplate, a cytoplasmic complex that represents the assembly platform for the tail. 8 The T6SS tail is structurally, evolutionarily and functionally similar to the contractile tails of 9 bacteriophages. We have shown that TssA docks to the membrane complex, recruits the 10 baseplate complex and initiates and coordinates the polymerization of the inner tube with that 11 of the sheath. Here, we review these recent findings, discuss the variations within TssA-like 12 proteins, speculate on the role of EAEC TssA in T6SS biogenesis and propose future research 13 perspectives. 


\section{Introduction}

2 The Type VI secretion system (T6SS) is a multiprotein machine widespread in

3 Proteobacteria and Bacteroidetes and responsible for the transport and delivery of toxins into

4 recipient cells (Figure 1) [1-3]. The T6SS targets both eukaryotic and prokaryotic cells and

5 therefore participates to pathogenesis towards plant, animal or human cells, as well as to inter-

6 bacterial competition [4-10]. Indeed, the T6SS anti-bacterial activity is responsible for re-

7 shaping bacterial communities providing growth and colonization advantages [6,11-18]. The

8 effectors are deleterious enzymes (peptidoglycan hydrolases, phospholipases, DNases, etc)

9 that are delivered into recipient cells as cargo by binding to components of the tail tube/spike

10 complex, which is propelled by a contractile mechanism [6-8,19-21]. This tail structure is

11 evolutionarily, structurally and functionally related to tails of contractile bacteriophages or of

12 R-pyocins [22-28]. It is composed of an inner tube made by stacked Hcp hexamers and tipped

13 by the VgrG trimeric spike complex (Figure 1B)[29,30]. The tail is surrounded by a sheath,

14 made of TssB and TssC subunits, that is assembled in an extended conformation that stores

15 mechanical energy necessary for its contraction (Figure 1B)[31-33]. The assembly of the

16 T6SS tail is controlled by the baseplate, a structure composed of the VgrG spike and the TssE,

17 TssF, TssG and TssK proteins [34-36]. The baseplate is anchored to the TssJLM transenvelope complex, the first T6SS element to be assembled (Figure 1B) [35,37-42]. The TssJLM membrane complex therefore constitutes the docking station for the tail and has been proposed to serve as channel for the passage of the tail tube/spike complex that is propelled

21 during sheath contraction [38]. 
Recent data using fluorescence microscopy approaches have provided insights onto

2 the biogenesis pathway of the T6SS. The assembly proceeds inward, from the outer

3 membrane to the cytoplasm: the membrane complex is assembled first, prior to the

4 recruitment of the baseplate and polymerization of the cytoplasmic tail $[35,38,43]$ (Figure 2).

\section{The TssJLM subunits assemble a large trans-envelope complex}

The T6SS membrane complex is composed of the TssJ, TssL and TssM proteins [44].

7 Tss J is an outer membrane lipoprotein [45,46] whereas TssL and TssM are both inner 8 membrane proteins $[39,47,48]$. TssL is inserted in the inner membrane via a C-terminal

9 transmembrane helix [48] whereas TssM possesses three transmembrane helices, helices 2 10 and 3 delimitating a $30-\mathrm{kDa}$ cytoplasmic domain bearing a NTPase fold [39,47]. The third transmembrane helix of TssM is followed by a large periplasmic domain that mediates interaction with TssJ $[38,46]$. Purification of the enteroaggregative E. coli T6SS membrane complex defined that it has a size of 1.7 MDa and comprises ten copies of each subunit [38].

14 The TssJLM complex has a 5-fold symmetry, and is constituted of five dimers of 15 heterotrimeric complexes [38]. Fluorescence microscopy analyses of GFP fusions to TssM and TssL have provided evidence that TssJ positions first and then sequentially recruits TssM and TssL (Figure 2A, blue inset; Figure 2B)[38]. In some cases, proper assembly of the T6SS membrane complex requires attachment to the peptidoglycan layer and the local degradation 19 of the cell wall [44, 49-51].

The T6SS tail is a mosaic structure that includes elements co-opted from diverse phages functional fusion between phage hub and spike proteins; TssE is homologous to a phage wedge component conserved in bacteriophages T4 (gp25), P2 (gpW) and Mu (Mup46); TssF 
1 and TssG share homologies with two phage wedge proteins: Mup47 and Mup48 of phage Mu,

2 and gpJ and gpI of phage P2 whereas TssK is a mosaic protein with a N-terminal domain

3 structurally and functionally similar to siphophage receptor-binding shoulder domains

$4 \quad[28,29,34-36,40,52-56]$. While they have diverse origins, the TssE, $-\mathrm{F},-\mathrm{G}$ and $-\mathrm{K}$ proteins

5 have been shown to form a stable complex [34-36]. Once assembled, the T6SS baseplate

6 complex is recruited to the TssJLM complex via multiple interactions involving the TssL and

7 TssM cytoplasmic domains and the TssE, $-\mathrm{G}$ and $-\mathrm{K}$ proteins (Figure 2A, pink inset; Figure

8 2B)[35,37,39,40,57]. In enteroaggregative E. coli, the recruitment of the baseplate complex

9 also requires the TssA subunit that binds to the TssJM complex prior to the recruitment of

10 TssL [41]. Then, the tail polymerizes onto the baseplate, with the recruitment of Hcp

11 hexamers and TssBC strands at the distal end of the growing structure (Figure

12 2) $[30,35,43,58,59]$. It has been proposed that the assembly of the inner tube is coordinated

13 with that of the sheath, the recruitment of an Hcp hexamer immediately preceding that of

14 TssBC [30]. Based on biochemical and fluorescence microscopy evidence, it has been

15 recently proposed that the EAEC TssA protein coordinates the assembly of the inner tube

16 with that of the sheath [41]. Hence, by interacting with the membrane complex, recruiting the

17 baseplate and coordinating tube/sheath polymerization, TssA is a central component of the

18 EAEC T6SS.

\section{TssA-like proteins come into different flavours: baseplate component or cap protein}

We have proposed that the EAEC TssA protein is involved in the different stages of

T6SS biogenesis but plays a major role by priming and coordinating the polymerization of the 
1 structural component of the baseplate per se [42]. However, while TssA has been shown to be

2 essential for T6SS function in EAEC and other strains such as Vibrio cholerae and

3 Agrobacterium tumefaciens [41], the P. aeruginosa TssA1 protein is dispensable for T6SS

4 function [42], suggesting that these two proteins play distinct roles. Worth noticing, while the

5 EAEC TssA and $P$. aeruginosa TssA1 proteins share a similar N-terminal domain of 6 unknown function (ImpA_N; PF06812), they have very distinct C-terminal extensions. The

7 TssA C-terminal domain (VasJ domain, PF16989) is structurally arranged as a hexaflexagon

8 [41] whereas the TssA1 C-terminal domain shares secondary structure homologies with gp6,

9 a subunit of the T4 phage baseplate (Figure 3A) [42]. In addition these two proteins have 10 distinct partners (Table 1)[41,42]. While TssA interacts with the different T6SS sub11 complexes, TssA1 interacts with baseplate and tube/sheath components and is predicted to be at the interface between the two sub-complexes (Figure 3B) [42]. Hence, TssA and TssA1 present different C-terminal extensions, do not share functional properties and should not be

14 confused. Interestingly, a third class of Tss A-like proteins, comprising a N-terminal ImpA 15 domain with a distinct C-terminal extension, is encoded within a limited number of T6SS 16 gene clusters. The C-terminal extensions of these proteins bear a hydrophobic region that may serve as membrane anchor and a domain of unknown function (VasL, PF12486) (Figure 3). Because this class of TssA-like proteins is likely to be an accessory component of the T6SS, we named it TagA (Type VI secretion accessory gene with Imp스 domain). We will focus this discussion on the EAEC T6SS, and more specifically on the role of TssA.

\section{TssA participates to the different T6SS assembly stages}

23 We have reported that TssA binds to the TssJM complex, prior to TssL recruitment and 24 polymerization to form the trans-envelope channel. TssA then binds to the baseplate and 
1 coordinates the assembly of the inner tube with that of the sheath. The structure of the

2 dodecameric TssA complex showed that it has a 6-arm starfish structure. The central core has

3 an hexaflexagon structure, comprising 6 triangles connected at the periphery by interactions

4 between two $\alpha$-helices from two adjacent monomers. Protein-protein interaction studies have

5 provided evidence that the central core interacts with Hcp hexamers whereas the arms interact

6 with the sheath. Interestingly, molecular modeling experiments showed that TssA can dock to

7 the sheath under the extended conformation but not to the contracted sheath [41]. We thus

8 proposed that by binding to the rigid inner tube via the central core, the TssA arms participate

9 to maintaining the sheath under the extended, metastable, conformation [41] (Figure 2B). The

10 role of TssA at each stage of the T6SS biogenesis pathway is discussed below.

11 TssA binds to the TssJLM membrane complex

The first partner of TssA is the membrane complex. Pull-down and fluorescence microscopy experiments have shown that TssA binds the TssJM complex, prior to the recruitment of TssL [41]. Two questions arise from these observations: how TssA interacts with the membrane proteins? How can we reconcile the symmetry mismatch between the 5fold symmetry TssJLM complex and the 6-fold symmetry TssA complex?

TssA interaction with TssJLM proteins. The assembly of the TssJLM membrane complex starts with the positioning of the TssJ outer membrane lipoprotein and the recruitment of TssM. The order of the last two steps, i.e., recruitment of TssL and polymerization to form the trans-envelope channel, remains unknown [38]. TssA is recruited to the TssJM complex. However, protein-protein interaction studies did not report contacts between TssA and the cytoplasmic loop connecting TssM helices 2 and 3 [41]. One may hypothesize that (i) TssA interacts to the cytoplasmic N-terminal 15-residue segment of TssM, (ii) TssA binding requires a combination of the cytoplasmic loop and $\mathrm{N}$-terminal segment, (iii) due to the lack 
1 of constraints imposed by the transmembrane helices, the isolated TssM cytoplasmic loop is

2 not in the proper conformation, or (iv) TssJ binding to TssM induces a conformational change

3 in the TssM cytoplasmic loop.

4 TssJLM-TssA symmetry mismatch. The TssJ, $-\mathrm{L}$ and $-\mathrm{M}$ were overproduced and the

5 TssJLM complex purified from E. coli K-12 cells. Negative strain electron microscopy

6 analyses further demonstrated that the TssJLM complex has a 5-fold symmetry (C5) [38]. By

7 contrast, TssA has a 6-fold symmetry (D6) [41]. How two objects with different symmetry

8 can accommodate [60]? Interestingly, it is well known in the phage field that symmetry

9 mismatches exist between the pentagonal head vertex and the dodecameric portal/connector

10 [61-63]. This symmetry mismatch has been proposed to be important for DNA packaging into

11 the head $[61,62,64]$. A similar situation may take place in the case of the T6SS membrane and

12 TssA/baseplate complexes. However, trying to dock the TssA electron microscopy structure

13 to the cytoplasmic part of the TssJLM complex using Chimera [65] did not generate a

14 reasonable model. Alternatively, TssA being recruited prior to TssL in vivo, one may imagine

15 that TssA acts as a chaperone and by controlling the polymerization of the TssJLM complex

16 forces the symmetrization to a 6-fold symmetry. To gain further insights onto TssJLM-A

17 complex formation, we generated a 6-fold symmetry model of the TssJLM complex. One fifth

18 of the TssJLM electron microscopy map was isolated and a 6-fold symmetry was applied to

19 this fragment (Figure 4). Interestingly, docking of TssA to the 6-fold symmetry TssJLM

20 complex resulted in a reasonable fit of the TssA arms between the densities assigned to the

21 TssL dimers (Figure 4). In addition, when comparing this model to the shape of the particles

22 obtained for TssJLM alone and for the co-expressed TssJLM and TssA complex, we observed

23 that the shape of the TssJLM-A particle fits well the generated 6-fold symmetry model

24 (Figure 5). Specifically, the "emerged" part of TssA in the model is comparable to the width

25 and thickness of its counterpart in the particles (Figure 5). Thus, it remains possible that TssA 
1 serves as a chaperone that would bind to TssJLM and stabilize a dodecamer with a 6-fold symmetry instead of the thermodynamically more stable isolated TssJLM decamer with a 5fold symmetry. Purification and imaging the TssJLM-A complexes, or imaging the TssJLM complex directly in enteroaggregative $E$. coli cells may provide critical information regarding the native symmetry of the T6SS membrane complex.

\section{TssA binds to the baseplate}

TssA does not remain attached to the TssJLM complex but rather binds the baseplate and then is positioned at the distal end of the tail during tail extension [41]. The first step of

9 TssA migration is the replacement of TssA by the baseplate at the TssJLM cytoplasmic base.

10 It has been proposed that the baseplate assembles in the cytosol, independently of the membrane complex but that it is stabilized when docked to the TssJLM complex [35]. One may hypothesize that the affinity of the TssJLM complex for the baseplate is higher than its affinity for TssA. Hence, the assembled baseplate should progressively displace the TssJLMTssA complex towards a TssJLM-baseplate complex.

\section{TssA coordinates assembly of the tail tube and tail sheath}

In absence of TssA, the Hcp hexamers do not properly stack on each other [35] and the sheath does not extend [41]. In addition, TssA remains at the distal end of the sheath during the extension process [41]. Based on these observations, we have suggested that TssA coordinates the assembly of the inner tube with that of the sheath [41]. This model agrees with recent data that showed that the sheath is growing by the distal end, i.e., sheath subunits are incorporated at the tip of the tubular structure [59]. Different models could be proposed on how TssA incorporates new subunits (Figure 6). In the "exchange" model (Figure 6A), a new 
1 TssA complex, loaded with the Hcp-TssBC complex is exchanged with the TssA located at

2 the distal end of the growing tail. A new row is then added. The "flip" model (Figure 6B)

3 takes into account the D6 symmetry of the TssA complex, which exhibits two identical sides

4 with identical surfaces. Once bound to the distal end of the growing tail, the free side of the

5 TssA complex recruits an Hcp-TssBC complex and then flips allowing the incorporation of a

6 new row. In the "cap" model (Figure 6C), the triangles of the central core of TssA are

7 displaced to open a lumen allowing the passage of an Hcp hexamer. Then the TssA arms

8 recruit, position and polymerize TssBC subunits, such as the flagellar cap does with flagellins

$9 \quad[66,67]$. Although movements of the TssA central core triangles have not been evidenced, we

10 favor the "cap" model as the arms will maintain the sheath under the extended conformation,

11 whereas the "exchange" and "flip" models imply dissociation of TssA from the distal end of 12 the tail.

TssA maintains the tail sheath under an extended conformation

How the sheath is maintained under the extended, high-energy, conformation? This question remains unsolved. We propose that TssA plays a key role in this process. The TssBC sheath is attached to the baseplate and to the Tss A arms [41]. Via its central core, TssA is bound to the Hcp tube, which represents a rigid structure between the baseplate and TssA. Thus the length of the inner tube controls the stretching of the sheath. In this model, all the energy of sheath extension is hold by the TssA arms, which is unlikely. It is highly probable that other forces are required to maintain the sheath under the extended conformation. Because polymerization of inner tube hexamers immediately precedes polymerization of sheath row, it is suggested that the inner tube serves as template for sheath polymerization

24 [30]. A similar assembly process has been proposed for the bacteriophage T4 tail [68]. 
1 Interestingly, the external surface of Hcp hexamers comprises charged residues and we may

2 hypothesize that charge interactions between the outer surface of the tube and the inner

3 surface of the sheath also contribute to the stabilization of the sheath in the extended

4 conformation. Such a charge complementarity between tube and sheath exists in the case of

5 R-pyocins [69].

6 TssA remains attached to the sheath distal end during contraction

In contractile bacteriophages, the contraction of the sheath is triggered by structural

8 transitions in the baseplate that are induced by the attachment of the long fibers to the prey

9 cell surface. Thus, sheath contraction is initiated at the baseplate level. If the mechanism is

10 similar in the case of the T6SS, TssA is in contact with the last row of sheath that contracts.

11 Docking simulations have shown that the TssA arms fit well with the sheath modeled in the

12 extended conformation (Figure 7) whereas cannot accommodate to the contracted sheath [41].

13 This modeling is in agreement with the observation that TssA is no co-purified with

14 contracted sheath in Vibrio cholerae [31,70]. By connecting the last sheath row to the tube,

15 TssA prevents energy dissipation during sheath contraction and allows tube propeling, before 16 being released.

\section{Conclusions and outlook}

Recent studies have highlighted that TssA is necessary at various stages of the T6SS assembly pathway: TssA binds the membrane complex and may have a chaperone role to control its assembly. It is involved in the recruitment of the baseplate and then primes and coordinates tail tube/sheath extension. TssA also participates to maintaining the sheath under the extended conformation. Finally, by connecting the distal end of the tube with the sheath 
1 until the last row of sheath contracts, TssA permits proper propulsion of the tube/spike. Many

2 of these aspects remain hypothetical but further experiments will likely provide details on the

3 role of TssA during T6SS biogenesis. Purification and high-resolution imaging of the

4 TssJLM-A complex will provide insights onto TssA docking to the MC and on the potential

5 symmetry mismatch. A better characterization of different TssA, TssA1 and TagA proteins

6 will help to better understand their contribution to T6SS biogenesis as well as to highlight

7 their functional and structural differences. Finally high-resolution fluorescence microscopy

8 technologies will provide information on how the T6SS tail assembles and whether TssA

9 remains associated with the sheath during contraction.

\section{Acknowledgements}

11 We thank the members of the Cascales, Cambillau/Roussel, Lloubès, Bouveret and Sturgis research groups for insightful discussions. This work was supported by the CNRS, the AixMarseille Université and grants from the Agence Nationale de la Recherche (ANR-10-JCJC1303-03 and ANR-14-CE14-0006-02). A.Z. was a recipient of a doctoral fellowship from the French Ministry of Research and a end-of-thesis fellowship from the Fondation pour la Recherche Médicale (FDT20140931060). The authors declare no conflict of interest.

\section{References}

[1] Silverman JM, Brunet YR, Cascales E, Mougous JD. 2012. Structure and regulation of the type VI secretion system. Annu Rev Microbiol. 66:453-72.

[2] Boyer F, Fichant G, Berthod J, Vandenbrouck Y, Attree I. 2009. Dissecting the bacterial type VI secretion system by a genome wide in silico analysis: what can be learned from available microbial genomic resources? BMC Genomics. 10:104.

[3] Russell AB, Wexler AG, Harding BN, Whitney JC, Bohn AJ, Goo YA, Tran BQ, Barry NA, Zheng H, Peterson SB, Chou S, Gonen T, Goodlett DR, Goodman AL, Mougous JD. 2014. A type VI secretion-related pathway in Bacteroidetes mediates interbacterial antagonism. Cell Host Microbe. 16:227-36. 
[4] Pukatzki S, Ma AT, Sturtevant D, Krastins B, Sarracino D, Nelson WC, Heidelberg JF, Mekalanos JJ. 2006. Identification of a conserved bacterial protein secretion system in Vibrio cholerae using the Dictyostelium host model system. Proc Natl Acad Sci U S A. 103:1528-33.

[5] Hood RD, Singh P, Hsu F, Güvener T, Carl MA, Trinidad RR, Silverman JM, Ohlson BB, Hicks KG, Plemel RL, Li M, Schwarz S, Wang WY, Merz AJ, Goodlett DR, Mougous JD. 2010. A type VI secretion system of Pseudomonas aeruginosa targets a toxin to bacteria. Cell Host Microbe. 7:25-37.

[6] Russell AB, Peterson SB, Mougous JD. 2014. Type VI secretion system effectors: poisons with a purpose. Nat Rev Microbiol. 12:137-48.

[7] Durand E, Cambillau C, Cascales E, Journet L. 2014. VgrG, Tae, Tle, and beyond: the versatile arsenal of Type VI secretion effectors. Trends Microbiol. 22:498-507.

[8] Alcoforado Diniz J, Liu YC, Coulthurst SJ. 2015. Molecular weaponry: diverse effectors delivered by the Type VI secretion system. Cell Microbiol. 17:1742-51.

[9] Hachani A, Wood TE, Filloux A. 2016. Type VI secretion and anti-host effectors. Curr Opin Microbiol. 29:81-93.

[10] Hood RD, Peterson SB, Mougous JD. 2017. From striking out to striking gold: discovering that Type VI secretion targets bacteria. Cell Host Microbe. 21:286-289.

[11] Kapitein N, Mogk A. 2013. Deadly syringes: type VI secretion system activities in pathogenicity and interbacterial competition. Curr Opin Microbiol. 16:52-8.

[12] Fu Y, Waldor MK, Mekalanos JJ. 2013. Tn-Seq analysis of Vibrio cholerae intestinal colonization reveals a role for T6SS-mediated antibacterial activity in the host. Cell Host Microbe. 14:652-63.

[13] Sana TG, Flaugnatti N, Lugo KA, Lam LH, Jacobson A, Baylot V, Durand E, Journet L, Cascales E, Monack DM. 2016. Salmonella Typhimurium utilizes a T6SS-mediated antibacterial weapon to establish in the host gut. Proc Natl Acad Sci U S A. 113:E5044-51.

[14] Wexler AG, Bao Y, Whitney JC, Bobay LM, Xavier JB, Schofield WB, Barry NA, Russell AB, Tran BQ, Goo YA, Goodlett DR, Ochman H, Mougous JD, Goodman AL. 2016. Human symbionts inject and neutralize antibacterial toxins to persist in the gut. Proc Natl Acad Sci US A. 113:3639-44.

[15] Chatzidaki-Livanis M, Geva-Zatorsky N, Comstock LE. 2016. Bacteroides fragilis type VI secretion systems use novel effector and immunity proteins to antagonize human gut Bacteroidales species. Proc Natl Acad Sci U S A. 113:3627-32. 
1 [16] Hecht AL, Casterline BW, Earley ZM, Goo YA, Goodlett DR, Bubeck Wardenburg J. 2016. Strain competition restricts colonization of an enteric pathogen and prevents colitis. EMBO Rep. 17:1281-91.

4

[17] Anderson MC, Vonaesch P, Saffarian A, Marteyn BS, Sansonetti PJ. 2017. Shigella sonnei encodes a functional T6SS used for interbacterial competition and niche occupancy. Cell Host Microbe. 21:769-776.

[18] Sana TG, Lugo KA, Monack DM. 2017. T6SS: the bacterial "fight club" in the host gut. PLoS Pathog. 13:e1006325.

[19] Benz J, Meinhart A. 2014. Antibacterial effector/immunity systems: it's just the tip of the iceberg. Curr Opin Microbiol. 17:1-10.

[20] Bleves S, Sana TG, Voulhoux R. 2014. The target cell genus does not matter. Trends Microbiol. 22:304-6.

[21] Bleves S. 2016. Game of trans-kingdom effectors. Trends Microbiol. 24:773-4.

[22] Cascales E, Cambillau C. 2012. Structural biology of type VI secretion systems. Philos Trans R Soc Lond B Biol Sci. 367:1102-11.

[23] Coulthurst SJ. 2013. The Type VI secretion system - a widespread and versatile cell targeting system. Res Microbiol. 164:640-54.

[24] Zoued A, Brunet YR, Durand E, Aschtgen MS, Logger L, Douzi B, Journet L, Cambillau C, Cascales E. 2014. Architecture and assembly of the Type VI secretion system. Biochim Biophys Acta. 1843:1664-73.

[25] Ho BT, Dong TG, Mekalanos JJ. 2014. A view to a kill: the bacterial type VI secretion system. Cell Host Microbe. 15:9-21.

[22] Basler M. 2015. Type VI secretion system: secretion by a contractile nanomachine. Philos Trans R Soc Lond B Biol Sci. 370:1679.

[27] Cianfanelli FR, Monlezun L, Coulthurst SJ. 2016. Aim, Load, Fire: The Type VI Secretion System, a Bacterial Nanoweapon. Trends Microbiol. 24:51-62.

[28] Büttner CR, Wu Y, Maxwell KL, Davidson AR. 2016. Baseplate assembly of phage Mu: Defining the conserved core components of contractile-tailed phages and related bacterial systems. Proc Natl Acad Sci U S A. 113:10174-9.

[29] Leiman PG, Basler M, Ramagopal UA, Bonanno JB, Sauder JM, Pukatzki S, Burley SK, Almo SC, Mekalanos JJ. 2009. Type VI secretion apparatus and phage tail-associated protein complexes share a common evolutionary origin. Proc Natl Acad Sci U S A. 106:4154-9. 
[30] Brunet YR, Hénin J, Celia H, Cascales E. 2014. Type VI secretion and bacteriophage tail tubes share a common assembly pathway. EMBO Rep. 15:315-21.

[31] Basler M, Pilhofer M, Henderson GP, Jensen GJ, Mekalanos JJ. 2012. Type VI secretion requires a dynamic contractile phage tail-like structure. Nature. 483:182-6.

[32] Kube S, Kapitein N, Zimniak T, Herzog F, Mogk A, Wendler P. 2014. Structure of the VipA/B type VI secretion complex suggests a contraction-state-specific recycling mechanism. Cell Rep. 8:20-30.

[33] Kudryashev M, Wang RY, Brackmann M, Scherer S, Maier T, Baker D, DiMaio F, Stahlberg H, Egelman EH, Basler M. 2015. Structure of the type VI secretion system contractile sheath. Cell. 160:952-62.

[34] English G, Byron O, Cianfanelli FR, Prescott AR, Coulthurst SJ. 2014. Biochemical analysis of TssK, a core component of the bacterial Type VI secretion system, reveals distinct oligomeric states of TssK and identifies a TssK-TssFG subcomplex. Biochem J. 461:291-304.

[35] Brunet YR, Zoued A, Boyer F, Douzi B, Cascales E. 2015. The Type VI secretion TssEFGKVgrG phage-like baseplate is recruited to the TssJLM membrane complex via multiple contacts and serves as assembly platform for tail tube/sheath polymerization. PLoS Genet. 11:e1005545.

[36] Taylor NM, Prokhorov NS, Guerrero-Ferreira RC, Shneider MM, Browning C, Goldie KN, Stahlberg H, Leiman PG. 2016. Structure of the T4 baseplate and its function in triggering sheath contraction. Nature. 533:346-52.

[37] Zoued A, Durand E, Bebeacua C, Brunet YR, Douzi B, Cambillau C, Cascales E, Journet L. 2013. TssK is a trimeric cytoplasmic protein interacting with components of both phage-like and membrane anchoring complexes of the type VI secretion system. J Biol Chem. 288:27031-41.

[38] Durand E, Nguyen VS, Zoued A, Logger L, Péhau-Arnaudet G, Aschtgen MS, Spinelli S, Desmyter A, Bardiaux B, Dujeancourt A, Roussel A, Cambillau C, Cascales E, Fronzes R. 2015. Biogenesis and structure of a type VI secretion membrane core complex. Nature. 523:55560.

[39] Logger L, Aschtgen MS, Guérin M, Cascales E, Durand E. 2016. Molecular dissection of the interface between the Type VI secretion TssM cytoplasmic domain and the TssG baseplate component. J Mol Biol. 428:4424-4437.

[40] Nguyen VS, Logger L, Spinelli S, Legrand P, Huyen Pham TT, Nhung Trinh TT, Cherrak Y, Zoued A, Desmyter A, Durand E, Roussel A, Kellenberger C, Cascales E, Cambillau C. 2017. Type VI secretion TssK baseplate protein exhibits structural similarities with phage receptorbinding proteins and evolved to bind the membrane complex. Nat Microbiol. 2: 17103. 
[41] Zoued A, Durand E, Brunet YR, Spinelli S, Douzi B, Guzzo M, Flaugnatti N, Legrand P, Journet L, Fronzes R, Mignot T, Cambillau C, Cascales E. 2016. Priming and polymerization of a bacterial contractile tail structure. Nature. 531:59-63.

[42] Planamente S, Salih O, Manoli E, Albesa-Jové D, Freemont PS, Filloux A. 2016. TssA forms a gp6-like ring attached to the type VI secretion sheath. EMBO J. 35:1613-27.

[43] Gerc AJ, Diepold A, Trunk K, Porter M, Rickman C, Armitage JP, Stanley-Wall NR,

[45] Aschtgen MS, Bernard CS, De Bentzmann S, Lloubès R, Cascales E. 2008. SciN is an outer

[46] Felisberto-Rodrigues C, Durand E, Aschtgen MS, Blangy S, Ortiz-Lombardia M, Douzi B, Cambillau C, Cascales E. 2011. Towards a structural comprehension of bacterial type VI secretion systems: characterization of the TssJ-TssM complex of an Escherichia coli pathovar. PLoS Pathog. 7:e1002386.

[47] Ma LS, Lin JS, Lai EM. 2009. An IcmF family protein, ImpLM, is an integral inner membrane protein interacting with ImpKL, and its walker a motif is required for type VI secretion systemmediated Hcp secretion in Agrobacterium tumefaciens. J Bacteriol. 191:4316-29.

[48] Aschtgen MS, Zoued A, Lloubès R, Journet L, Cascales E. 2012. The C-tail anchored TssL subunit, an essential protein of the enteroaggregative Escherichia coli Sci-1 Type VI secretion system, is inserted by YidC. Microbiologyopen. 1:71-82.

[49] Aschtgen MS, Thomas MS, Cascales E. 2010. Anchoring the type VI secretion system to the cell wall: TssL, TagL, TagP... what else? Virulence. 1:535-40.

[50] Weber BS, Hennon SW, Wright MS, Scott NE, de Berardinis V, Foster LJ, Ayala JA, Adams MD, Feldman MF. 2016. Genetic dissection of the Type VI secretion system in Acinetobacter and identification of a novel peptidoglycan hydrolase, TagX, required for its biogenesis. MBio. 7: pii: e01253-16.

[51] Santin YG, Cascales E. 2016. Domestication of a housekeeping transglycosylase for assembly of a Type VI secretion system. EMBO Rep. pii: e201643206. 
[52] Pukatzki S, Ma AT, Sturtevant D, Krastins B, Sarracino D, Nelson WC, Heidelberg JF, Mekalanos JJ. 2006. Identification of a conserved bacterial protein secretion system in Vibrio cholerae using the Dictyostelium host model system. Proc Natl Acad Sci U S A. 103:18-33.

[53] Pukatzki S, Ma AT, Revel AT, Sturtevant D, Mekalanos JJ. 2007. Type VI secretion system translocates a phage tail spike-like protein into target cells where it cross-links actin. Proc Natl Acad Sci U S A. 104:15508-13.

[54] Bingle LE, Bailey CM, Pallen MJ. 2008. Type VI secretion: a beginner's guide. Curr Opin Microbiol. 11:3-8.

[55] Cascales E. 2008. The type VI secretion toolkit. EMBO Rep. 9:735-41.

[56] Lossi NS, Dajani R, Freemont P, Filloux A. 2011. Structure-function analysis of HsiF, a gp25like component of the type VI secretion system, in Pseudomonas aeruginosa. Microbiology. 157:3292-305.

[57] Zoued A, Cassaro CJ, Durand E, Douzi B, España AP, Cambillau C, Journet L, Cascales E. 2016. Structure-function analysis of the TssL cytoplasmic domain reveals a new interaction between the Type VI secretion baseplate and membrane complexes. J Mol Biol. 428:4413-4423.

[58] Brunet YR, Espinosa L, Harchouni S, Mignot T, Cascales E. 2013. Imaging type VI secretion-mediated bacterial killing. Cell Rep. 3:36-41.

[59] Vettiger A, Winter J, Lin L, Basler M. 2017. The type VI secretion sheath assembles at the distal end from the membrane anchor. Nat Commun. 8:16088.

[60] Goodsell DS, Olson AJ. 2000. Structural symmetry and protein function. Annu Rev Biophys Biomol Struct. 29:105-53.

[61] Hendrix RW. 1978. Symmetry mismatch and DNA packaging in large bacteriophages. Proc Natl Acad Sci U S A. 75:4779-83.

[62] Simpson AA, Tao Y, Leiman PG, Badasso MO, He Y, Jardine PJ, Olson NH, Morais MC, Grimes S, Anderson DL, Baker TS, Rossmann MG. 2000. Structure of the bacteriophage phi29 DNA packaging motor. Nature. 408:745-50.

[63] Zhao H, Speir JA, Matsui T, Lin Z, Liang L, Lynn AY, Varnado B, Weiss TM, Tang L. 2016. Structure of a bacterial virus DNA-injection protein complex reveals a decameric assembly with a constricted molecular channel. PLoS One. 11:e0149337.

[64] Simpson AA, Leiman PG, Tao Y, He Y, Badasso MO, Jardine PJ, Anderson DL, Rossmann MG. 2001. Structure determination of the head-tail connector of bacteriophage phi29. Acta Crystallogr D Biol Crystallogr. 57:1260-9. 
[65] Pettersen EF, Goddard TD, Huang CC, Couch GS, Greenblatt DM, Meng EC, Ferrin TE. 2004. UCSF Chimera--a visualization system for exploratory research and analysis. J Comput Chem. 25:1605-12.

[66] Yonekura K, Maki S, Morgan DG, DeRosier DJ, Vonderviszt F, Imada K, Namba K. 2000.

The bacterial flagellar cap as the rotary promoter of flagellin self-assembly. Science. 290:2148-52.

[67] Yonekura K, Maki-Yonekura S, Namba K. 2002. Growth mechanism of the bacterial flagellar

[68] Arisaka F, Tschopp J, Van Driel R, Engel J. 1979. Reassembly of the bacteriophage T4 tail from the core-baseplate and the monomeric sheath protein P18: a co-operative association process.

[69] Ge P, Scholl D, Leiman PG, Yu X, Miller JF, Zhou ZH. 2015. Atomic structures of a bactericidal contractile nanotube in its pre- and postcontraction states. Nat Struct Mol Biol. 22:377-

[71] Durand E, Zoued A, Spinelli S, Watson PJ, Aschtgen MS, Journet L, Cambillau C, 82.

[70] Bönemann G, Pietrosiuk A, Diemand A, Zentgraf H, Mogk A. 2009. Remodelling of VipA/VipB tubules by $\mathrm{ClpV}$-mediated threading is crucial for type VI protein secretion. EMBOJ. 28:315-25.

\section{Legend to Figures}

Figure 1. Type VI secretion system: genes and general architecture. Schematic representations of the genes involved in the assembly of the T6SS (A) and the T6SS architecture (B). The genes and their corresponding proteins are indicated with the same colour code, and are categorized in sub-complexes (membrane complex, tail comprising the baseplate complex, spike, tube, sheath and cap). Except for Hcp, VgrG, PAAR and ClpV, T6SS genes are indicated with the 1-letter Tss nomenclature (e.g., L corresponds to tssL). (OM, outer membrane; PG, peptidoglycan; IM, inner membrane; cyto, cytoplasm). 
1 biogenesis starts with the assembly of the membrane complex (MC; blue frame) and the 2 recruitment of the baseplate (BP) that has been proposed to be assembled independently (pink 3 frame), prior to polymerization of the tail tube/sheath, contraction and recycling of the sheath.

4 The TssA (indicated in red) protein is recruited to the TssJM complex and primes tail tube/sheath polymerization and coordinates assembly of the sheath with that of the tube. (B)

6 Schematic representation of the T6SS biogenesis pathway highlighting the role of TssA (red).

7 TssA is recruited to the TssJM complex, prior to TssL and is required for recruitment of the 8 baseplate. TssA coordinates tail tube/sheath assembly and is released at the end of the sheath 9 contraction. Note that in this scheme, contraction of the sheath is initiated at the baseplate, a hypothesis that is based on data on contractile bacteriophages.

Figure 3. Architecture and localization of TssA-like proteins. (A) Architecture of the three classes of TssA-like proteins, named TssA, TssA1 and TagA. The conserved ImpA_N domain (pfam accession PF06812) is shown in red whereas the C-terminal extensions are shown in colour. Interesting features are indicated (T6SS_VasJ, domain of unknown function [pfam accession PF16989] [blue]; gp6, bacteriophage gp6 homology region [orange]; HR, hydrophobic region [grey]; VasL, domain of unknown function [pfam accession PF12486] [green]). (B) Localizations of the TssA (left), TssA1 (middle) and TagA (right) proteins. The TssA-like proteins are shown in red with their suggested localizations. No information is available for the TagA protein.

Figure 4. 6-fold symmetrization of the TssJLM complex and TssA docking simulations. Side (A), Bottom (B) and cut-away (C, D) views of the docking simulations of the TssA dodecamer electron microscopy structure (purple [41], EMD-3982) to the base of the 6-fold symmetrized TssJLM complex (grey). The positions of the $\mathrm{C}$ and $\mathrm{D}$ cut sections are indicated in panel B. In panel (B), the localization of one TssL dimer density $[38,71]$ is indicated.

Figure 5. Comparison of the 6-fold symmetry TssJLM-TssA model with a electron microscopy particle. The model of the 6-fold symmetrized TssJLM-TssA complex (A) is compared to a TssJLM-TssA (B, C [41]) and TssJLM (D, E [38]) particles. Scale bars are 10 $\mathrm{nm}$.

Figure 6. Schematic models for TssA-mediated recruitment and polymerization of tube/sheath subunits. Exchange (A), flip (B) and cap (C) models proposed to explain the mode of action of TssA (red) during tail tube/sheath (blue) extension. A new TssA molecule 
1 is indicated in orange whereas the newly incorporated tube/sheath building block is indicated 2 in green.

3 Figure 7. Simulation modelling of TssA bound to the extended sheath. Side view of the 4 docking simulation of the TssA dodecamer electron microscopy structure (purple) bound to 5 the distal end of the T6SS sheath modelled in the extended conformation (grey). 
A
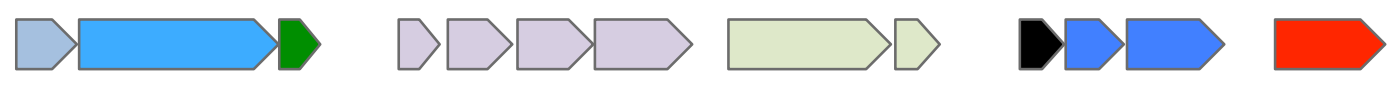

L M J

E F G K

VgrG PAAR Hcp B

C

A
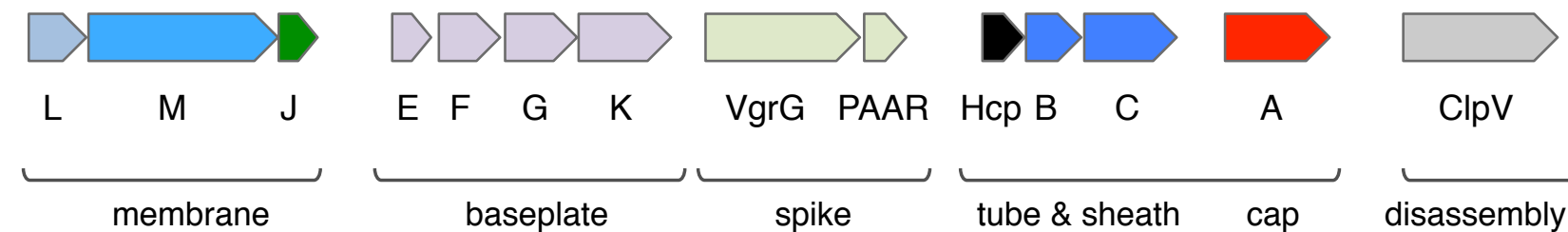

complex

(MC)

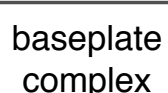

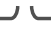

spike

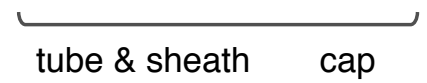

complex

(BP)

tail

B

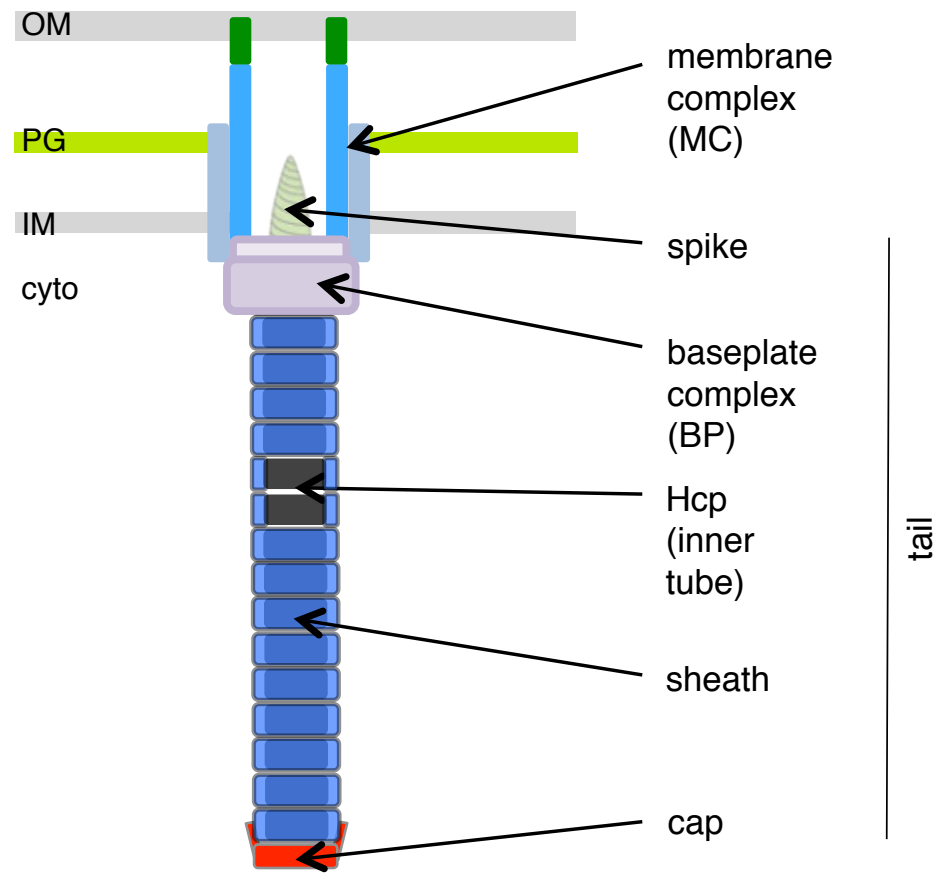




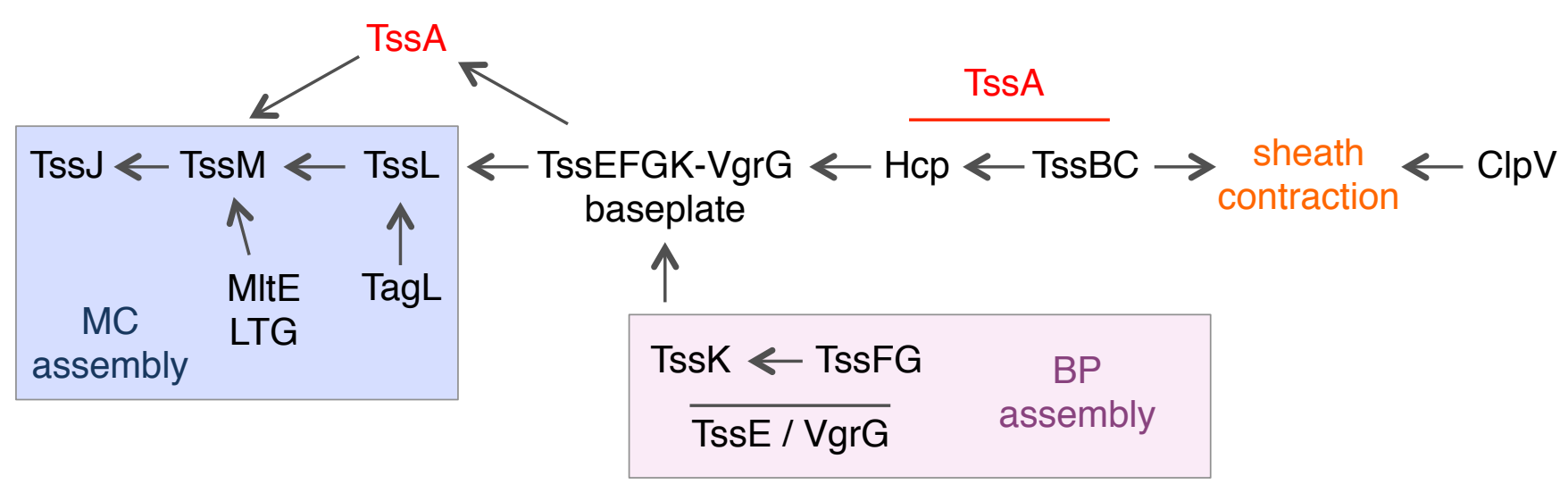

B

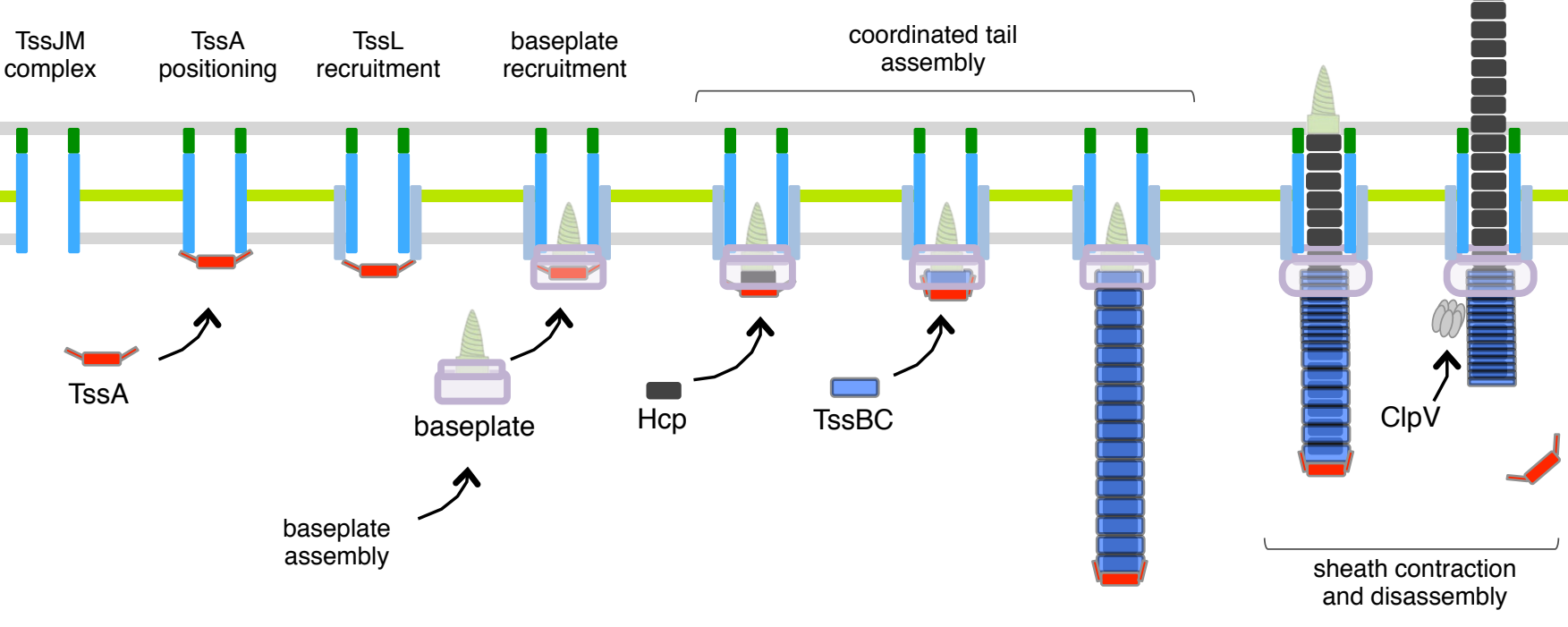


A

TssA

TssA1

$$
\text { ImpA_N }
$$

gp6

TagA

$$
\text { ImpA_N }
$$

HR

\section{VasL}

B

$$
\text { TssA }
$$

TssA1

TagA

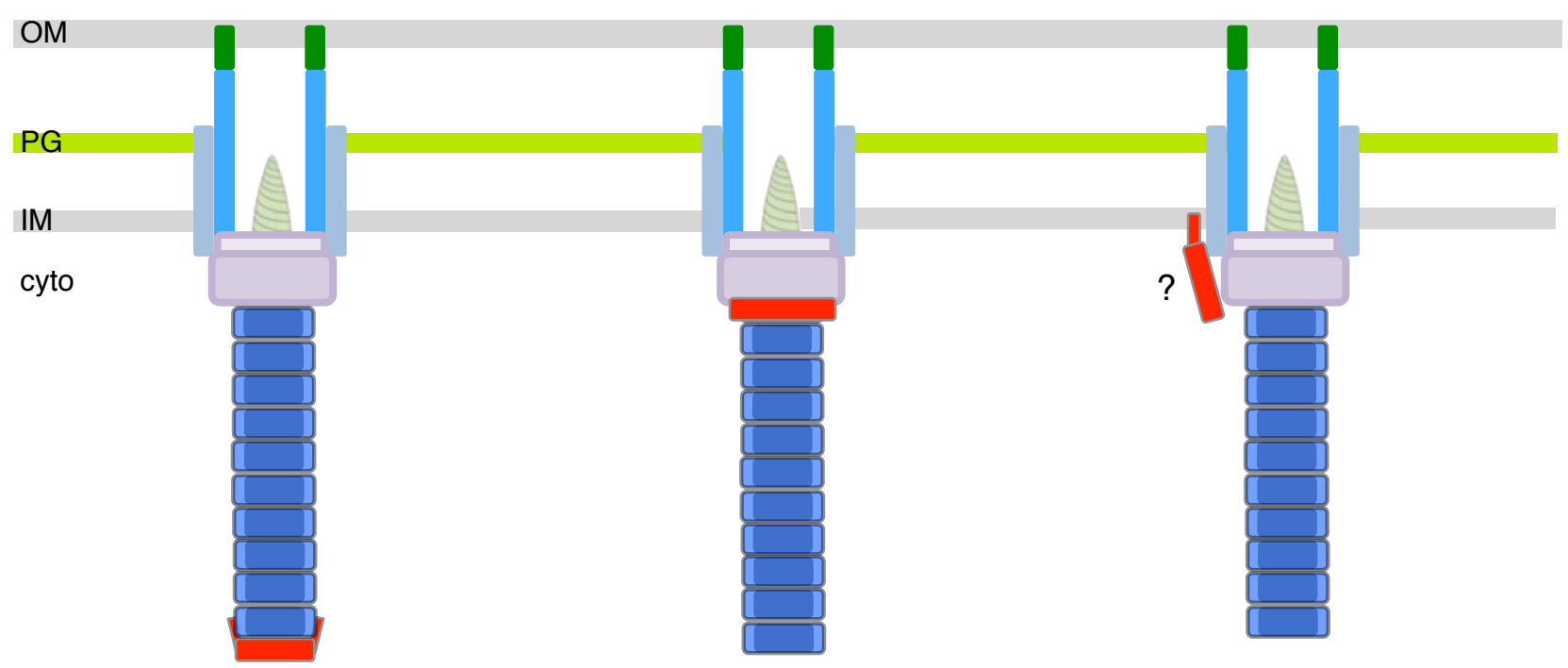

Figure 3 


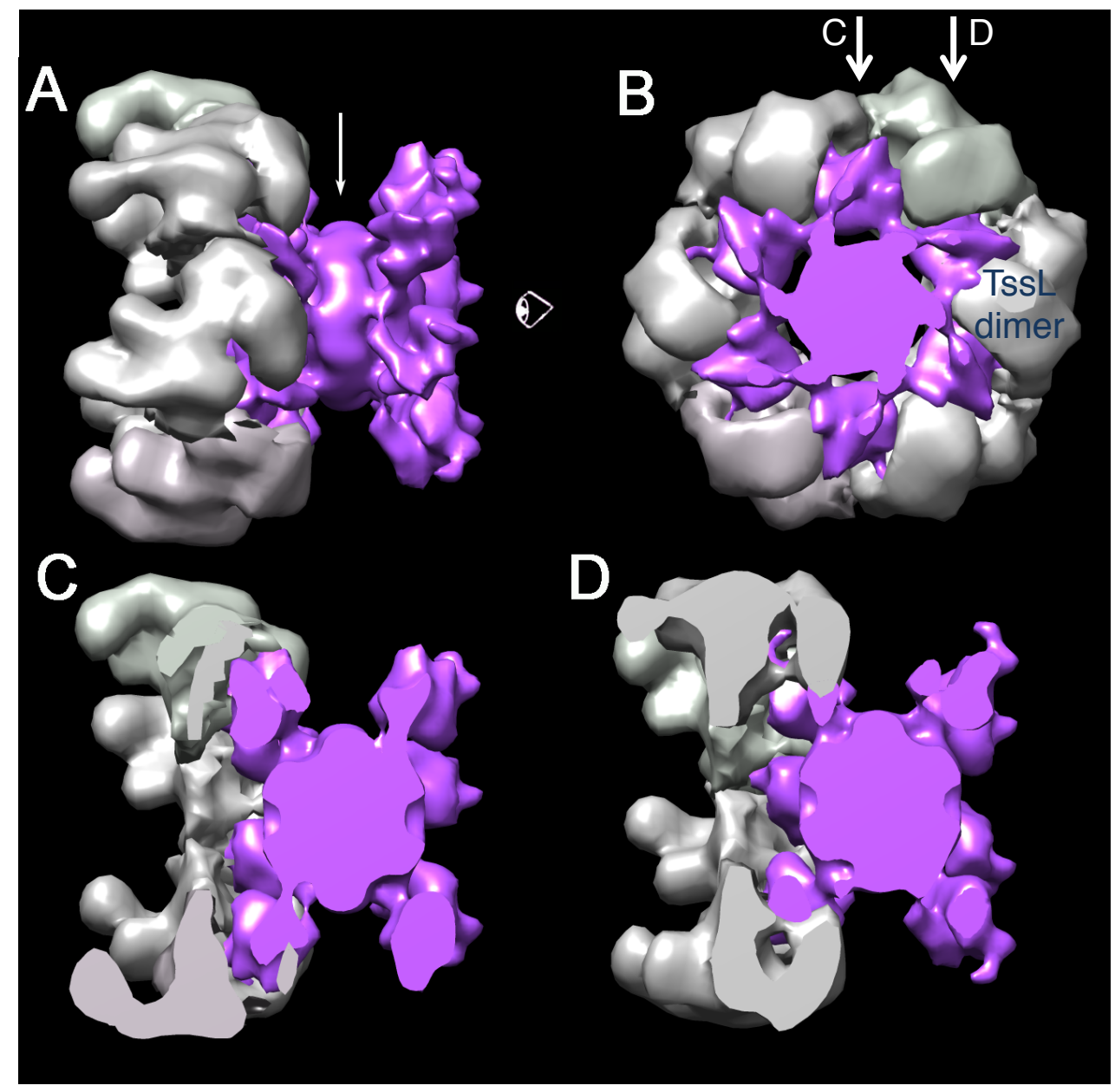

Figure 4 


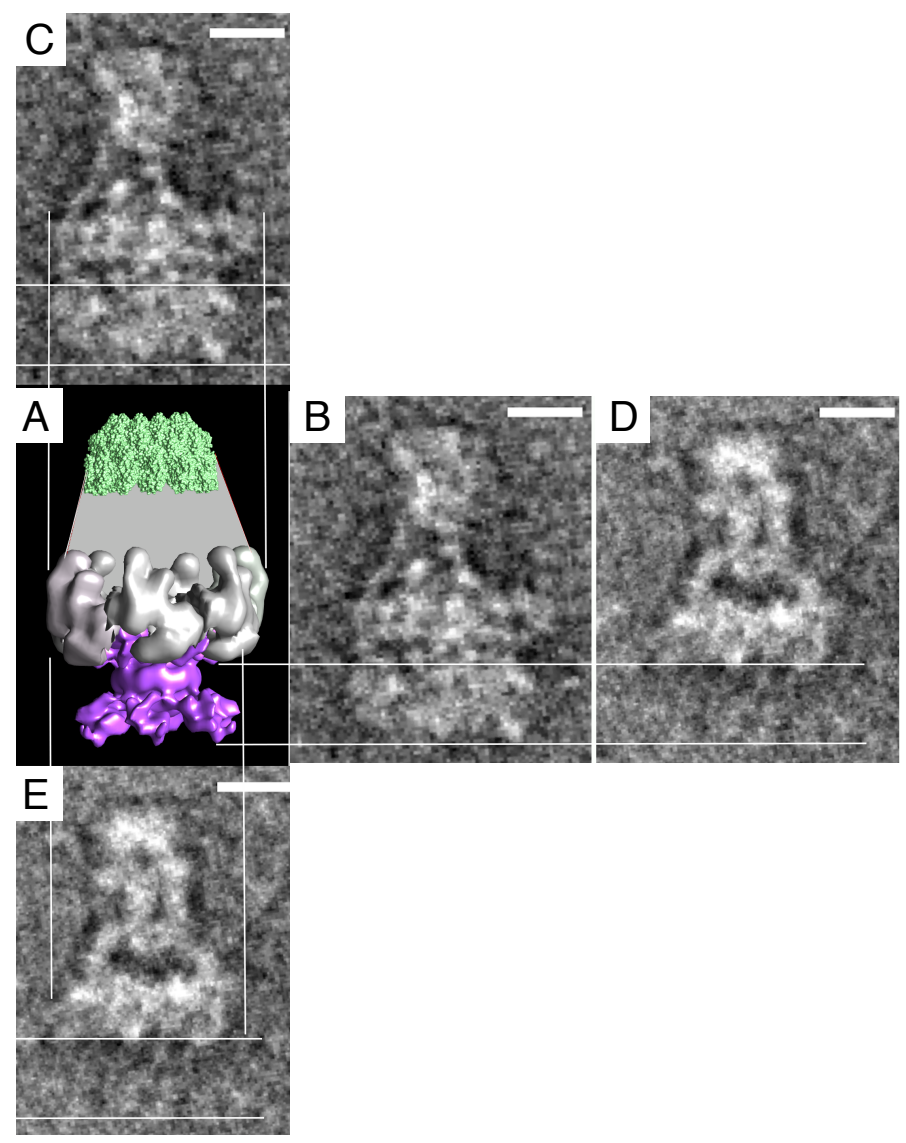

Figure 5 
A exchange

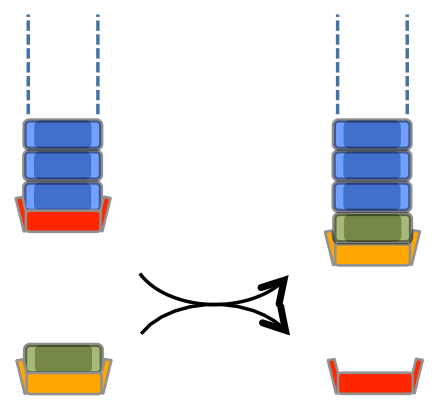

B flip

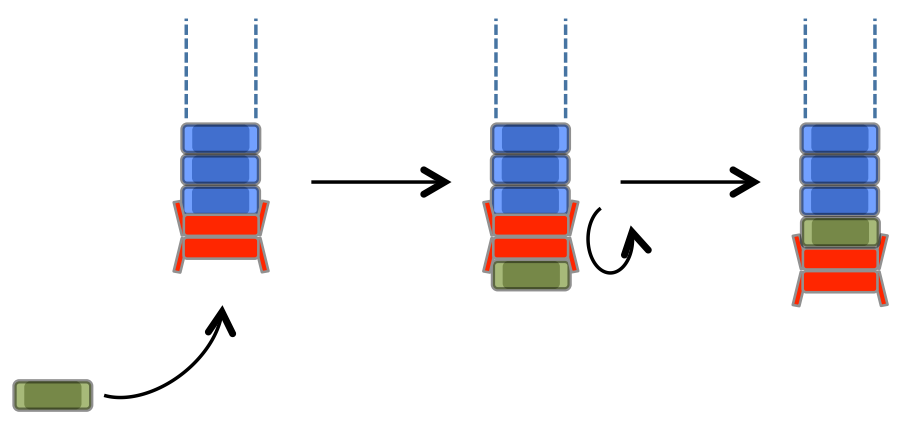

C cap

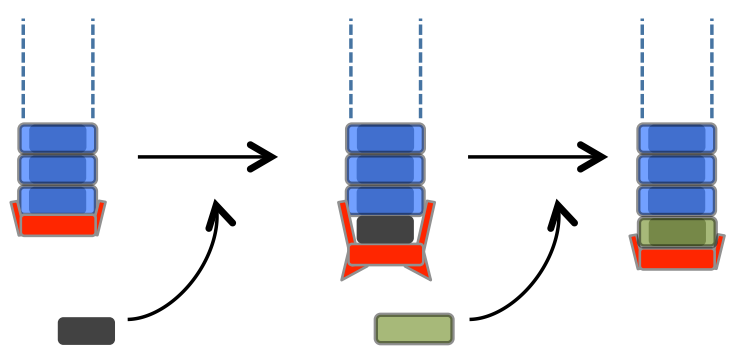

$$
\text { Hcp }
$$

Figure 6 


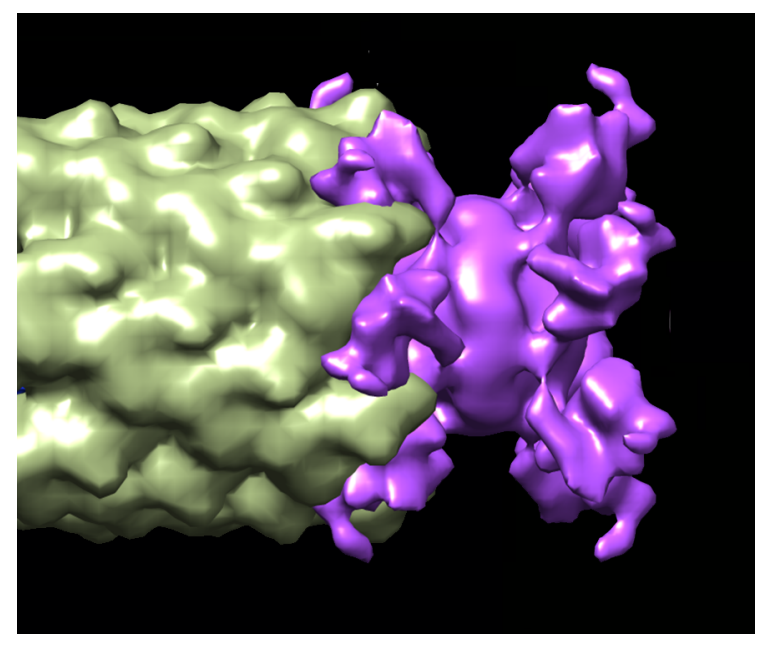

Figure 7 